\title{
CALCULATED ENERGY DISTRIBUTIONS FOR SN II
}

\author{
P.H. Hauschildt, W. Spies, R. Wehrse \\ Institut für Theoretische Astrophysik, Universität Heidelberg \\ Im Neuenheimer Feld 561, D-6900 Heidelberg \\ G. Shaviv \\ Dept. of Physics, Technion, Israel Institute of Technology \\ IL-32000 Haifa
}

\section{ABSTRACT}

We have calculated a large grid of hydrogen-rich supernova photospheres, in which radii, effective temperatures, density profiles, and expansion velocities have been varied. Spherical geometry, radiative equilibrium and LTE level populations are assumed. In the quasi-exact radiative transfer, the dilution of the radiation field, and scattering as well as absorption (by all relevant continuous processes and up to 150000 lines in some models) are accurately considered. Good agreement can be obtained with the UV and IR spectra of supernovae 1979C, 1980K, and $1987 \mathrm{~A}$ as observed during the coasting phase. Potential methods of parameter determinations for SN II are briefly discussed.

Since supernovae of type II are believed to be explosions of massive stars with large hydrogen-rich shells (see e.g. Woosley and Weaver, 1986), the spectra of these objects contain important information on the abundances of the elements of the parent stars (and therefore implicitly on the abundances of the parent galaxy) during the first days up to a few weeks after the outburst and at later times information on the amount and the composition of the processed matter returned to the interstellar medium. In addition, the atmospheric parameters can be used to determine the distance to the object with a minimum of assumptions by means of the Baade-Wesselink method. Since - due to the very high brightness of SNe II - the data can be obtained for a very large volume of space, it is highly desirable to model in detail the photospheres of these objects. However, until recently realistic models were inhibited by the required complexity of the radiative transfer (spherical geometry, differential expansion, dominance of scattering, line blanketing etc.).

In order to obtain an overview on the significance of these effects and on the influence of the parameters on the emergent fluxes we have calculated a large grid of spherical hydrogen-rich supernova photospheres with the following assumptions: ( $i$ ) the radial density distribution is given by a power law (exponents $n=5 \ldots 14$ ), (ii) the expansion velocities are proportional to the radial distance (the values at absorption 
optical depths of unity are varied between $5.10^{3}$ and $15.10^{3} \mathrm{~km} \mathrm{~s}^{-1}$, , (iii) the level populations follow Boltzmann distributions of the local temperature, (iv) the temperature stratifications are derived from the condition of radiative equilibrium. In the non-grey quasiexact radiative transfer all relevant opacity sources for the continuum (cf. Hauschildt et al., 1987) and in some models up to 150000 individual lines are taken into account. We consider ranges of effective temperature $6000 \leq T_{\text {eff }} \leq 15000 \mathrm{~K}$ and radius $10^{13} \leq \mathrm{R} \leq 10^{15} \mathrm{~cm}$. The composition is solar, except for two sets in which the metal content is reduced to $1 / 3$ and $1 / 10$ solar.

The resulting energy distributions show that for $\mathrm{T}_{\text {eff }}>10^{4} \mathrm{~K}$ line blanketing is of minor importance (even in the UV), but for lower temperatures it is highly significant both for the spectrum and for the temperature stratification. In most wavelength ranges the fluxes are strongly influenced simultaneously by $n, R$, and $T_{\text {eff }}$, i.e. a change in one parameter can be compensated by changes in the other ones. However, rather accurate determinations of $T_{\text {eff }}$ seem to be possible from the slope in the red and infrared range. The radii and density parameters should be derived from a simultaneous fit of the Blamer discontinuity and the flux gradient in the ultraviolet. It seems difficult to obtain accurate estimates for the abundances of individual metals from UV and optical spectra because usually many lines are blended and most lines have absorption and emission components. But from such features the expansion velocities can be determined rather reliably:

Comparison of our calculated fluxes with the observed spectra of the SNe $1979 \mathrm{C}$ and $1980 \mathrm{~K}$ shows that good agreement can be obtained for the UV and the IR during the coasting phase (see e.g. Hauschildt et al., 1987). In the visible a good fit is possible as long as the Balmer lines are weak indicating NLTE effects for hydrogen in the outer line forming layers as expected (Höflich et al., 1986). For the heavily blanketed SN 1987A the main features in the UV can be reproduced for metallicities $1 / 3$ to $1 / 1$ solar; however, details indicate that the relative abundances in the irongroup differ from the solar values.

\section{ACKNOWLEDGMENT}

This work was supported by the Deutsche Forschungsgemeinschaft (SFB 328). 


\section{REFERENCES}

Hauschildt, P., Spies, W., Wehrse, R., Shaviv, G.: 1987, Proc. Eso Workshop "SN 1987A", Garching, in press

Höflich, P., Wehrse, R., Shaviv, G.: 1986, Astron. Astrophys. 163,105

Spies, W.: 1987, Synthetische Spektren für supernovae vom Typ II, Diplomarbeit Heidelberg

Spies, W., Hauschildt, P., Wehrse, R., Baschek, B., Shaviv, G.: in Nuclear Astrophysics, Lecture Notes in Physics 287,316, Springer, 1987

Woosley, S.E., Weaver, T.A.: 1986, Ann. Rev. Astron. Astrophys. 24, 205 